\title{
Flagged Grothendieck polynomials
}

\author{
Tomoo Matsumura $^{1}$
}

Received: 16 January 2017 / Accepted: 19 September 2018 / Published online: 27 September 2018

(c) Springer Science+Business Media, LLC, part of Springer Nature 2018

\begin{abstract}
We show that the flagged Grothendieck polynomials defined as generating functions of flagged set-valued tableaux of Knutson et al. (J Reine Angew Math 630:1-31, 2009) can be expressed by a Jacobi-Trudi-type determinant formula generalizing the work of Hudson-Matsumura (Eur J Comb 70:190-201 2018). We also introduce the flagged skew Grothendieck polynomials in these two expressions and show that they coincide.
\end{abstract}

Keywords Grothendieck polynomials · flagged set-valued tableaux · vexillary permutations $\cdot$ Jacobi-Trudi formula

Mathematics Subject Classification 05E05 $\cdot 14 \mathrm{M} 15 \cdot 13 \mathrm{D} 15$

\section{Introduction}

Lascoux-Schützenberger $[16,18]$ introduced the Grothendieck polynomials to represent the $K$-theory classes of the structure sheaves of Schubert varieties, and Fomin-Kirillov [7,8] gave their combinatorial description in terms of pipe dreams or rc graphs. Knutson-Miller-Yong [15] expressed the Grothendieck polynomial associated with a vexillary permutation as the generating function of flagged set-valued tableaux, unifying the work of Wachs [20] on flagged tableaux and Buch [4] on setvalued tableaux. On the other hand, in the joint work [12] with Hudson, the author proved the Jacobi-Trudi-type formula for the vexillary Grothendieck polynomials in the context of degeneracy loci formulas, generalizing his joint work [10] with Hudson, Ikeda, and Naruse for the Grassmannian case (see also [1,11,19]).

Motivated by these results, we study the generating functions of flagged set-valued tableaux in general, beyond the ones given by vexillary permutations. We follow the

The author is supported by JSPS Grant-in-Aid for Young Scientists (B) 16K17584.

\footnotetext{
$\bowtie$ Tomoo Matsumura

matsumur@xmath.ous.ac.jp

1 Department of Applied Mathematics, Okayama University of Science, Okayama 700-0005, Japan
} 
works $[14,15]$ of Knutson-Miller-Yong. For a given partition $\lambda=\left(\lambda_{1} \geq \cdots \geq \lambda_{r}>\right.$ $0)$, a flagging $f=\left(f_{1}, \ldots, f_{r}\right)$ of $\lambda$ is a nondecreasing sequence of positive integers. A flagged set-valued tableau of shape $\lambda$ with a flagging $f$ is nothing but a set-valued tableaux of shape $\lambda$ of Buch [4] satisfying extra conditions that the numbers used in the $i$ th row are at most $f_{i}$ for all $i$. Let $\operatorname{FSVT}(\lambda, f)$ be the set of all flagged set-valued tableaux of shape $\lambda$ with the flagging $f$. Let $x=\left(x_{1}, x_{2}, \ldots\right)$ be a set of infinitely many indeterminants. We define the flagged Grothendieck polynomial $G_{\lambda, f}(x)$ by

$$
G_{\lambda, f}(x):=\sum_{T \in F \operatorname{SVT}(\lambda, f)} \beta^{|T|-|\lambda|} x^{T}
$$

where $x^{T}$ is the monomial in which the exponent of $x_{i}$ is the number of boxes in $T$ containing the integer $i, T$ is the total degree of $x^{T}$, and $|\lambda|=\lambda_{1}+\cdots+\lambda_{r}$.

The main goal of this paper is to show that the flagged Grothendieck polynomial $G_{\lambda, f}(x)$ is given by the following Jacobi-Trudi-type determinant formula (Theorem 1)

$$
G_{\lambda, f}(x)=\operatorname{det}\left(\sum_{s=0}^{\infty}\left(\begin{array}{c}
i-j \\
s
\end{array}\right) \beta^{s} G_{\lambda_{i}+j-i+s}^{\left[f_{i}\right]}(x)\right)_{1 \leq i, j \leq r} .
$$

Here, we define $G_{m}^{[p]}(x)$ for $m \in \mathbb{Z}$ by the generating function

$$
\sum_{m \in \mathbb{Z}} G_{m}^{[p]}(x) u^{m}=\frac{1}{1+\beta u^{-1}} \prod_{1 \leq i \leq p} \frac{1+\beta x_{i}}{1-x_{i} u},
$$

which geometrically correspond to the Segre classes of vector bundles (see [10]). Our result indeed extends the equivalence of the tableau formula of Knutson-MillerYong [15] and the determinant formula of Hudson-Matsumura [12] in the view of the fact that not all flagged Grothendieck polynomials are Grothendieck polynomials of Lascoux and Schützenberger. It is also worth noting that our proof of Theorem 1 is completely combinatorial and it does not depend on any result that is geometrically proved.

The flagged Grothendieck polynomials $G_{\lambda, f}(x)$ specialize to the flagged Schur polynomials at $\beta=0$. These polynomials were introduced by Lascoux and Schützenberger in [17] to identify Schubert polynomials associated with vexillary permutations. They are also generalizations of Schur polynomials and satisfy the generalized JacobiTrudi formula, due to Gessel and Wachs. In this paper, we closely follow Wachs' inductive proof in [20], which makes the use of the divided difference operators. In particular, our proof shows that the above determinant formula in terms of the one-row Grothendieck polynomials $G_{m}^{[p]}(x)$ behaves nicely under the action of those symmetrizing operators.

In Sect. 3, we give a combinatorial proof of Knutson-Miller-Yong's result and of the determinant formula in [12]. Namely, based on the results in Sect.2, we prove that the Grothendieck polynomial associated with a vexillary permutation is a flagged Grothendieck polynomial. We also show that any flagged Grothendieck polynomial can be obtained from a monomial by applying the divided difference operators. 
In Sect. 4, we introduce the flagged skew Grothendieck polynomials. Their tableaux and determinant expressions are natural extensions of the ones for the (row) flagged skew Schur functions studied by Wachs [20] in the view of the formulas of KnutsonMiller-Yong [15] and Hudson-Matsumura [12]. We show that those two expressions coincide.

It is worth pointing out that the formulas of Knutson-Miller-Yong and HudsonMatsumura are valid for double Grothendieck polynomials defined for two sets of variables. Thus it would be natural to extend our result to its double version (see the work [5] of Chen-Li-Louck for the flagged double Schur functions). This extension will be studied elsewhere.

After this work was completed, Anderson-Chen-Tarasca [2] showed that the double Grothendieck polynomials associated with 321-avoiding permutations are the double version of flagged skew Grothendieck polynomials defined by the determinant formula. See also [3] and [6] for the relations between the Schubert polynomials associated with 321-avoiding permutations and the flagged skew Schur polynomials.

\section{Flagged Grothendieck polynomials}

A partition is a nonincreasing finite sequence $\lambda=\left(\lambda_{1}, \ldots, \lambda_{r}\right)$ of positive integers, and $r$ is called its length. We often identify a partition $\lambda$ of length $r$ with its Young diagram $\left\{(i, j) \mid 1 \leq i \leq r, 1 \leq j \leq \lambda_{i}\right\}$ in English notation. We let $|\lambda|=\lambda_{1}+\cdots+\lambda_{r}$. A flagging $f$ of a partition $\lambda$ of length $r$ is a nondecreasing sequence $f=\left(f_{1}, \ldots, f_{r}\right)$ of positive integers. A flagged set-valued tableau $T$ of shape $\lambda$ with a flagging $f$ is a set-valued tableau of shape $\lambda$ such that each filling in the $i$ th row consists of the numbers not greater than $f_{i}$. Namely, $T$ fills each box at $(i, j)$ of $\lambda$ by a non-empty subset of $\left\{1, \ldots, f_{i}\right\}$ in such a way that the maximum number used at $(i, j)$ is at most the minimum number used at $(i, j+1)$ for $j+1 \leq \lambda_{i}$ and less than the minimum number used at $(i+1, j)$ for $j \leq \lambda_{i+1}$. In particular, if $f_{1}=\cdots=f_{r}$, then it coincides with the definition of set-valued tableaux defined by Buch in [4]. Knutson-MillerYong [15] studied flagged set-valued tableaux in the context of more abstract notion of tableau complexes where their flagging $f$ is not necessarily weakly increasing. We denote by $\operatorname{FSVT}(\lambda, f)$ the set of all flagged set-valued tableaux of shape $\lambda$ with the flagging $f$.

Example 1 For example, let $\lambda=(2,1)$ and $f=(2,4)$. Then $\operatorname{FSVT}(\lambda, f)$ contains tableaux such as

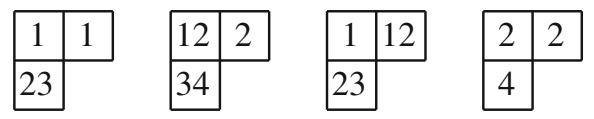

If we change $f$ to $f^{\prime}=(2,3)$, then $\operatorname{FSVT}\left(\lambda, f^{\prime}\right)$ does not contain the second and fourth tableaux.

Let $x=\left(x_{1}, x_{2}, \ldots\right)$ be the set of infinitely many variables. Let $\mathbb{Z}[\beta]$ be the polynomial ring of the variable $\beta$ where we set $\operatorname{deg} \beta=-1$. Let $\mathbb{Z}[\beta][x]$ and $\mathbb{Z}[\beta][[x]]$ 
be the ring of polynomials and of formal power series in $x$, respectively. For each tableaux $T$, we set $x^{T}$ to be the monomial in which the exponent of $x_{i}$ is the number of boxes in $T$ containing the integer $i$. We let $|T|$ be the total degree of $x^{T}$. For example, the monomials $x^{T}$ for the tableaux in Example 1 are $x_{1}^{2} x_{2} x_{3}, x_{1} x_{2}^{2} x_{3} x_{4}, x_{1}^{2} x_{2}^{2} x_{3}$, and $x_{2}^{2} x_{4}$, respectively.

We define the flagged Grothendieck polynomial associated with a partition $\lambda$ and a flagging $f$ by

$$
G_{\lambda, f}(x):=\sum_{T \in F S V T(\lambda, f)} \beta^{|T|-|\lambda|} x^{T} .
$$

We also define an element $\widetilde{G}_{\lambda, f}(x)$ of $\mathbb{Z}[\beta][[x]]$ by

$$
\widetilde{G}_{\lambda, f}(x):=\operatorname{det}\left(\sum_{s=0}^{\infty}\left(\begin{array}{c}
i-j \\
s
\end{array}\right) \beta^{s} G_{\lambda_{i}+j-i+s}^{\left[f_{i}\right]}(x)\right)_{1 \leq i, j \leq r},
$$

where $G_{m}^{[p]}(x) \in \mathbb{Z}[\beta][[x]]$ is defined by the generating function

$$
G^{[p]}(x ; u)=\sum_{m \in \mathbb{Z}} G_{m}^{[p]}(x) u^{m}=\frac{1}{1+\beta u^{-1}} \prod_{1 \leq i \leq p} \frac{1+\beta x_{i}}{1-x_{i} u}
$$

For the empty partition, we set both of the functions $G_{\lambda, f}(x)$ and $\widetilde{G}_{\lambda, f}(x)$ to be 1. We also remark that $G_{-m}^{[p]}=(-\beta)^{m}$ and $G_{m}^{[1]}=x_{1}^{m}$ for all integer $m \geq 0$, which follow from the direct computation.

At $\beta=0, G_{\lambda, f}(x)$ specializes to the flagged Schur polynomial of Wachs in [20], and $\widetilde{G}_{\lambda, f}(x)$ is nothing but the corresponding Jacobi-Trudi formula since $G_{m}^{[p]}(x)$ becomes the complete symmetric function of degree $m$.

The main goal of this section is to show that $G_{\lambda, f}(x)$ and $\widetilde{G}_{\lambda, f}(x)$ coincide (Theorem 1). We closely follow Wachs' proof of the analogous statement for the flagged Schur polynomials in [20]. The key for the proof is the action of the divided difference operators, which makes the induction proof possible.

\subsection{Divided difference operators and basic formulas}

We recall the definition of divided difference operators $(c f .[4,8])$ and show a few formulas that will be used in the proofs of the propositions and the main theorem to follow.

Let $S_{n}$ be the permutation group of the set $\{1, \ldots, n\}$. We have the action of $S_{n}$ on $\mathbb{Z}[\beta][[x]]$ permuting the variables. For example, let $s_{i}=(i, i+1)$ denote the $i$ th simple reflection transposition, i.e., $s_{i}(i)=i+1, s_{i}(i+1)=i$ and $s_{i}(j)=j$ for $j \neq i, i+1$, then $s_{i}(f(x))$ is defined by exchanging $x_{i}$ and $x_{i+1}$ in $f(x) \in \mathbb{Z}[\beta][[x]]$ and leave other variables unchanged. 
Definition 1 For an element $f(x)$ of $\mathbb{Z}[\beta][[x]]$, we define

$$
\pi_{i}(f(x)):=\frac{\left(1+\beta x_{i+1}\right) f(x)-\left(1+\beta x_{i}\right) s_{i}(f(x))}{x_{i}-x_{i+1}} .
$$

The following Leibniz rule can be checked by a direct computation: For $f(x), g(x) \in \mathbb{Z}[\beta][[x]]$, we have

$$
\pi_{i}(f g)=\pi_{i}(f) g+s_{i}(f) \pi_{i}(g)+\beta s_{i}(f) g .
$$

It is also easy to check directly that if $f(x)$ is symmetric in $x_{i}$ and $x_{i+1}$, then we have

$$
\pi_{i}\left(x_{i}^{k} f(x)\right)= \begin{cases}-\beta f(x) & (k=0) \\ \left(\sum_{s=0}^{k-1} x_{i}^{s} x_{i+1}^{k-1-s}+\beta \sum_{s=1}^{k-1} x_{i}^{s} x_{i+1}^{k-s}\right) f(x) & (k>0) .\end{cases}
$$

Lemma 1 For each $m \in \mathbb{Z}$ and $p \in \mathbb{Z}_{\geq 1}$, we have

$$
\pi_{i}\left(G_{m}^{[p]}(x)\right)= \begin{cases}G_{m-1}^{[p+1]}(x) & (i=p) \\ -\beta G_{m}^{[p]}(x) & (i \neq p)\end{cases}
$$

Proof If $i=p$, it follows from the identity $\pi_{p} G^{[p]}(x ; u)=u G^{[p+1]}(x ; u)$ which can be proved by a direct computation. If $i \neq p$, then $G_{m}^{[p]}(x)$ is symmetric in $x_{i}$ and $x_{i+1}$, and hence (2.5) implies the claim.

Lemma 2 If $f(x)$ is symmetric in $x_{p}$ and $x_{p+1}$, then we have $\pi_{p}\left(G_{m}^{[p]} f\right)=G_{m-1}^{[p+1]} f$.

Proof It follows from the Leibniz rule (2.4) and Lemma 1.

Lemma 3 For each $m \in \mathbb{Z}$ and $p \in \mathbb{Z}_{\geq 1}$, we have

$$
G_{m}^{[p]}-\frac{x_{1}}{1+\beta x_{1}} G_{m-1}^{[p]}-\frac{x_{1}}{1+\beta x_{1}} \beta G_{m}^{[p]}=\left.G_{m}^{[p]}\right|_{x_{1}=0}
$$

Proof It follows from the identity

$$
\sum_{m \in \mathbb{Z}}\left(G_{m}^{[p]}-\frac{x_{1}}{1+\beta x_{1}} G_{m-1}^{[p]}-\frac{x_{1}}{1+\beta x_{1}} \beta G_{m}^{[p]}\right) u^{m}=\left.G^{[p]}(x ; u)\right|_{x_{1}=0}
$$

which can be checked by a direct computation. 


\subsection{The main theorem}

We prove the main theorem (Theorem 1) below by induction based on the following two propositions.

Proposition 1 Let $\lambda$ be a partition of length $r$ with a flagging $f$. If $\lambda_{1}>\lambda_{2}$ and $f_{1}<f_{2}$ (or $\left.r=1\right)$, then we have

(i) $\pi_{f_{1}}\left(\widetilde{G}_{\lambda, f}\right)=\widetilde{G}_{\lambda^{\prime}, f^{\prime}}$,

(ii) $\pi_{f_{1}}\left(G_{\lambda, f}\right)=G_{\lambda^{\prime}, f^{\prime}}$,

where $\lambda^{\prime}=\left(\lambda_{1}-1, \lambda_{2}, \ldots, \lambda_{r}\right)$ and $f^{\prime}=\left(f_{1}+1, f_{2}, \ldots, f_{r}\right)$.

Proof For (i), we recall from $[10, \S 3.6]$ that we can write

$$
\widetilde{G}_{\lambda, f}=\sum_{\mathbf{s} \in \mathbb{Z}^{r}} a_{\mathbf{s}} G_{\lambda_{1}+s_{1}}^{\left[f_{1}\right]} \cdots G_{\lambda_{r}+s_{r}}^{\left[f_{r}\right]},
$$

where $a_{\mathbf{s}} \in \mathbb{Z}[\beta]$ is the coefficient of $t^{\mathbf{s}}$ in the Laurent series expansion

$$
\prod_{1 \leq i<j \leq r}\left(1-\bar{t}_{i} / \bar{t}_{j}\right)=\sum_{\mathbf{s}=\left(s_{1}, \ldots, s_{r}\right) \in \mathbb{Z}^{r}} a_{\mathbf{s}} t_{1}^{s_{1}} \cdots t_{r}^{s_{r}} .
$$

Here, we denoted $\bar{t}=\frac{-t}{1+\beta t}=-t \sum_{s \geq 0}(-\beta)^{s} t^{s}$. Since $f_{1}<f_{2}$, one can apply Lemma 2 to the expression (2.6) and obtain (i). Indeed, we have

$$
\begin{aligned}
\pi_{f_{1}}\left(\widetilde{G}_{\lambda, f}\right) & =\sum_{\mathbf{s} \in \mathbb{Z}^{r}} a_{\mathbf{s}} \pi_{f_{1}}\left(G_{\lambda_{1}+s_{1}}^{\left[f_{1}\right]} \cdots G_{\lambda_{r}+s_{r}}^{\left[f_{r}\right]}\right) \\
& =\sum_{\mathbf{s} \in \mathbb{Z}^{r}} a_{\mathbf{s}} G_{\lambda_{1}-1+s_{1}}^{\left[f_{1}+1\right]} G_{\lambda_{2}+s_{2}}^{\left[f_{2}\right]} \cdots G_{\lambda_{r}+s_{r}}^{\left[f_{r}\right]} \\
& =\widetilde{G}_{\lambda^{\prime}, f^{\prime}}
\end{aligned}
$$

Next we prove (ii). Let $t:=f_{1}$ and $t^{\prime}:=f_{1}+1$. Define an equivalence relation $\sim$ on $\operatorname{FSVT}(\lambda, f)$ as follows. For $T_{1}, T_{2} \in \operatorname{FSVT}(\lambda, f)$, let $T_{1} \sim T_{2}$ if

- the collection of boxes that contain either $t$ or $t^{\prime}$ is the same for $T_{1}$ and $T_{2}$;

- the box at $\left(1, \lambda_{1}\right)$ in both $T_{1}$ and $T_{2}$ contains only $t$ or contains entries other than $t$, provided it contains $t$.

We have

$$
G_{\lambda, f}=\sum_{\mathscr{A} \in F \operatorname{SVT}(\lambda, f) /}\left(\sum_{T \in \mathscr{A}} \beta^{|T|-|\lambda|} x^{T}\right) .
$$

Let $\mathscr{A}$ be the equivalence class whose tableaux have the configuration of $t$ and $t^{\prime}$ as shown in Fig. 1. 


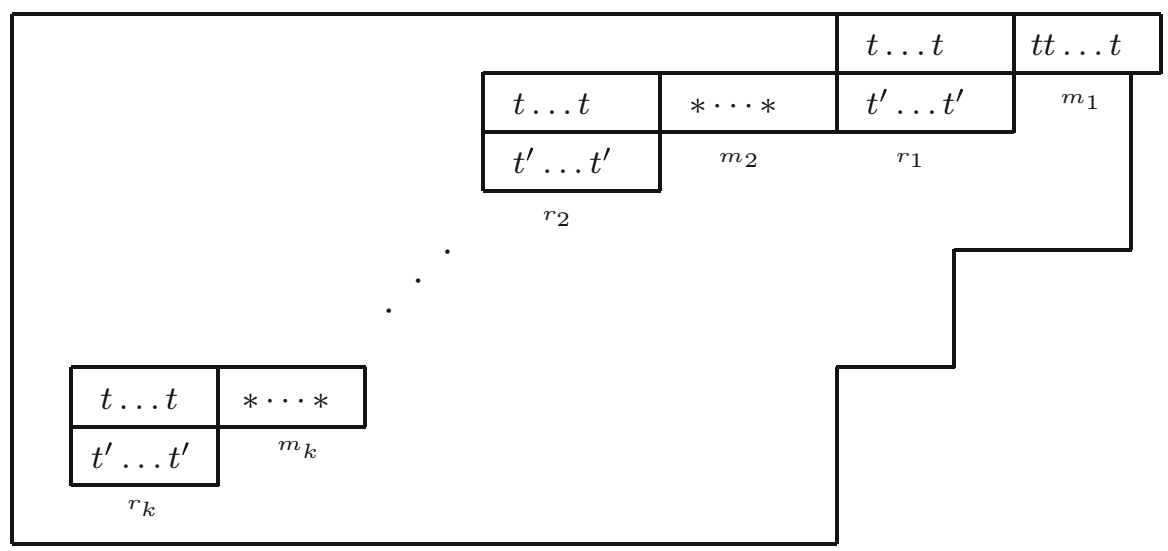

Fig. 1 Configuration of $t$ and $t^{\prime}$ for $\mathscr{A}$

Each rectangle with $*$ has $m_{i}$ boxes, and each box contains $t$ or $t^{\prime}$ so that the total number of entries $t$ and $t^{\prime}$ in the rectangle is $m_{i}$ or $m_{i}+1$ for $i \geq 2$. Note that $m_{i}$ and $r_{i}$ may be 0 and the rectangles in Fig. 1 may not be connected. Then we see that

$$
\begin{aligned}
& \sum_{T \in \mathscr{A}} \beta^{|T|-|\lambda|} x^{T} \\
& \quad=x_{t}^{m_{1}}\left(x_{t} x_{t^{\prime}}\right)^{r_{1}+\cdots+r_{k}}\left(\prod_{i=2}^{k}\left(\sum_{v=0}^{m_{i}} x_{t}^{v} x_{t^{\prime}}^{m_{i}-v}+\beta \sum_{v=1}^{m_{i}} x_{t}^{v} x_{t^{\prime}}^{m_{i}+1-v}\right)\right) R(\mathscr{A}),
\end{aligned}
$$

where $R(\mathscr{A})$ is the polynomial contributed from the entries other than $t$ and $t^{\prime}$. Let

$$
R^{\prime}(\mathscr{A}):=\left(\prod_{i=2}^{k}\left(\sum_{v=0}^{m_{i}} x_{t}^{v} x_{t^{\prime}}^{m_{i}-v}+\beta \sum_{v=1}^{m_{i}} x_{t}^{v} x_{t^{\prime}}^{m_{i}+1-v}\right)\right) R(\mathscr{A}) .
$$

Observe that the factor $\left(x_{t} x_{t^{\prime}}\right)^{r_{1}+\cdots+r_{k}} R^{\prime}(\mathscr{A})$ is symmetric in $x_{t}$ and $x_{t^{\prime}}$. Thus, by (2.5), if $m_{1}=0$, then we have $r_{1}=0$ and

$$
\pi_{t}\left(\sum_{T \in \mathscr{A}} \beta^{|T|-|\lambda|} x^{T}\right)=-\beta\left(x_{t} x_{t^{\prime}}\right)^{r_{2}+\cdots+r_{k}} R^{\prime}(\mathscr{A}),
$$

if $m_{1}=1$, we have

$$
\pi_{t}\left(\sum_{T \in \mathscr{A}} \beta^{|T|-|\lambda|} x^{T}\right)=\left(x_{t} x_{t^{\prime}}\right)^{r_{1}+\cdots+r_{k}} R^{\prime}(\mathscr{A})
$$


and if $m_{1} \geq 2$, we have

$$
\begin{aligned}
\pi_{t}\left(\sum_{T \in \mathscr{A}} \beta^{|T|-|\lambda|} x^{T}\right)= & \left(\sum_{s=0}^{m_{1}-1} x_{t}^{s} x_{t^{\prime}}^{m_{1}-1-s}+\beta \sum_{s=1}^{m_{1}-1} x_{t}^{s} x_{t^{\prime}}^{m_{1}-s}\right) \\
& \times\left(x_{t} x_{t+1}\right)^{r_{1}+\cdots+r_{k}} R^{\prime}(\mathscr{A}) .
\end{aligned}
$$

We consider the decomposition

$$
\operatorname{FSVT}(\lambda, f) / \sim=\mathcal{F}_{1} \sqcup \mathcal{F}_{2} \sqcup \mathcal{F}_{3} \sqcup \mathcal{F}_{4}
$$

where $\mathcal{F}_{1}, \ldots, \mathcal{F}_{4}$ are the sets of equivalence classes $\mathcal{A}$ with Fig. 1 , respectively, satisfying

(1) $m_{1}=0$ (so that $r_{1}=0$ ),

(2) $m_{1}=1$ and the box at $\left(1, \lambda_{1}\right)$ in $\lambda$ contains more than one entry (so that $r_{1}=0$ ),

(3) $m_{1}=1$ and the box at $\left(1, \lambda_{1}\right)$ in $\lambda$ contains only $t$,

(4) $m_{1} \geq 2$.

There is a bijection from $\mathcal{F}_{2}$ to $\mathcal{F}_{1}$ by sending $\mathcal{A}$ to $\mathcal{A}^{\prime}$ whose configuration is obtained by deleting $t$ from the box at $\left(1, \lambda_{1}\right)$ in the configuration of $\mathcal{A}$. Under this bijection, the tableaux in $\mathcal{A}$ and $\mathcal{A}^{\prime}$ correspond bijectively: $T \in \mathcal{A}$ maps to $T^{\prime} \in \mathcal{A}^{\prime}$ by deleting $t$ in the box at $\left(1, \lambda_{1}\right)$. This implies that, by the expressions (2.7) and (2.8) and the observation that $R(\mathcal{A})=\beta R\left(\mathcal{A}^{\prime}\right)$, we have

$$
\sum_{\mathscr{A} \in \mathcal{F}_{1} \sqcup \mathcal{F}_{2}} \pi_{t}\left(\sum_{T \in \mathscr{A}} \beta^{|T|-|\lambda|} x^{T}\right)=0 .
$$

Now consider the equivalence relation in $\operatorname{FSVT}\left(\lambda^{\prime}, f^{\prime}\right)$ defined by setting $T_{1}^{\prime} \sim T_{2}^{\prime}$ if the collection of boxes that contain either $t$ or $t^{\prime}$ is the same for $T_{1}^{\prime}$ and $T_{2}^{\prime}$. Let $\mathscr{A}^{\prime}$ be the equivalence class whose associated skew diagram of boxes containing $t$ or $t^{\prime}$ is as shown in Fig. 2 .

Consider the decomposition of $F S V T\left(\lambda^{\prime}, f^{\prime}\right) / \sim$ into $\mathcal{F}_{3}^{\prime}$ and $\mathcal{F}_{4}^{\prime}$ whose equivalence classes have configurations with $m_{1}=1$ and $m_{1} \geq 2$. There is a bijection from $\mathcal{F}_{3}$ to $\mathcal{F}_{3}^{\prime}$ sending $\mathcal{A}$ to $\mathcal{A}^{\prime}$ whose configuration is given by deleting the whole box at $\left(1, \lambda_{1}\right)$ together with the entry $t$. Also there is a bijection from $\mathcal{F}_{4}$ to $\mathcal{F}_{4}^{\prime}$ sending $\mathcal{A}$ to $\mathcal{A}^{\prime}$ whose configuration is given by deleting the whole box at $\left(1, \lambda_{1}\right)$ together with the entry $t$ and replacing $t$ by $*$ in the boxes from $\left(1, \lambda_{1}-m_{1}+1\right)$ to $\left(1, \lambda_{1}-1\right)$. Under this correspondence, if $\mathscr{A}^{\prime} \in \mathcal{F}_{3}^{\prime}$, then $\sum_{T \in \mathscr{A}^{\prime}} \beta^{|T|-|\lambda|} x^{T}$ is exactly the right-hand side of (2.8). If $\mathscr{A}^{\prime} \in \mathcal{F}_{4}^{\prime}$, then $\sum_{T \in \mathscr{A}^{\prime}} \beta^{|T|-|\lambda|} x^{T}$ is exactly the right-hand side of (2.9). Thus the desired identity holds.

Proposition 2 Let $\lambda$ be a partition of length $r$ with a flagging $f$. If $f_{1}=1$, then we have 


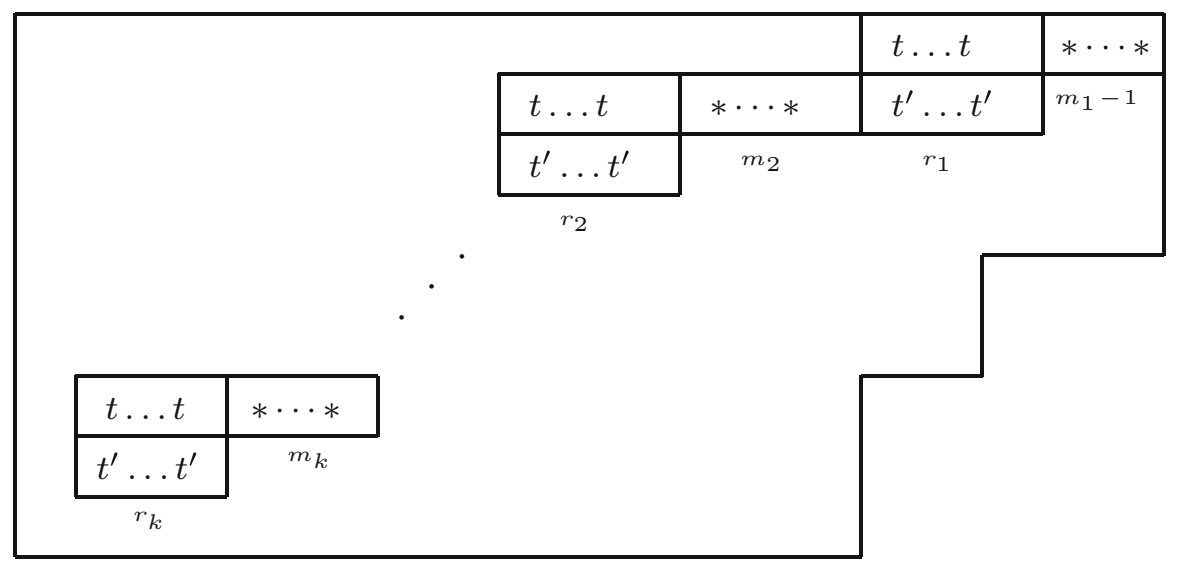

Fig. 2 Configuration of $t$ and $t^{\prime}$ for $\mathscr{A}^{\prime}$

(i) $\widetilde{G}_{\lambda, f}=x_{1}^{\lambda_{1}}\left(\left.\widetilde{G}_{\lambda^{\prime}, f^{\prime}}\right|_{x_{1}=0}\right)$,

(ii) $G_{\lambda, f}=x_{1}^{\lambda_{1}}\left(\left.G_{\lambda^{\prime}, f^{\prime}}\right|_{x_{1}=0}\right)$,

where $\lambda^{\prime}=\left(\lambda_{2}, \ldots, \lambda_{r}\right)$ and $f^{\prime}=\left(f_{2}, \ldots, f_{r}\right)$.

Proof First we observe that (ii) holds clearly since $f_{1}=1$. We prove (i). Since $f_{1}=1$, we see that the first row of the determinant for $\widetilde{G}_{\lambda, f}$ is

$$
\left(x_{1}^{\lambda_{1}}, x_{1}^{\lambda_{1}} \frac{x_{1}}{1+\beta x_{1}}, \ldots, x_{1}^{\lambda_{1}}\left(\frac{x_{1}}{1+\beta x_{1}}\right)^{r-1}\right)
$$

Indeed, since $G_{m}^{[1]}=x_{1}^{m}$ for $m \geq 0$, we have

$$
\sum_{s=0}^{\infty}\left(\begin{array}{c}
1-j \\
s
\end{array}\right) \beta^{s} G_{\lambda_{1}+j-1+s}^{\left[f_{1}\right]}(x)=x_{1}^{\lambda_{1}+j-1} \sum_{s=0}^{\infty}\left(\begin{array}{c}
1-j \\
s
\end{array}\right) \beta^{s} x_{1}^{s}=\frac{x_{1}^{\lambda_{1}+j-1}}{\left(1+\beta x_{1}\right)^{j-1}} .
$$

We do the column operation to $\widetilde{G}_{\lambda, f}$ by subtracting $\frac{x_{1}}{1+\beta x_{1}}$ times the $(j-1)$-st column from the $j$ th column for each $j=2, \ldots, r$ so that the first row becomes $\left(x_{1}^{\lambda_{1}}, 0, \ldots, 0\right)$. Then we observe that the $(i, j)$-entry for $i, j \geq 2$ equals to

$$
\begin{aligned}
& \sum_{s=0}^{\infty}\left(\begin{array}{c}
i-j \\
s
\end{array}\right) \beta^{s} G_{\lambda_{i}+j-i+s}^{\left[f_{i}\right]}-\frac{x_{1}}{1+\beta x_{1}} \sum_{s=0}^{\infty}\left(\begin{array}{c}
i-j+1 \\
s
\end{array}\right) \beta^{s} G_{\lambda_{i}+j-1-i+s}^{\left[f_{i}\right]} \\
& \quad=\sum_{s=0}^{\infty}\left(\begin{array}{c}
i-j \\
s
\end{array}\right) \beta^{s}\left(G_{\lambda_{i}+j-i+s}^{\left[f_{i}\right]}-\frac{x_{1}}{1+\beta x_{1}} G_{\lambda_{i}+j-1-i+s}^{\left[f_{i}\right]}-\frac{x_{1}}{1+\beta x_{1}} \beta G_{\lambda_{i}+j-i+s}^{\left[f_{i}\right]}\right) \\
& =\left.\left(\sum_{s=0}^{\infty}\left(\begin{array}{c}
i-j \\
s
\end{array}\right) \beta^{s} G_{\lambda_{i}+j-i+s}^{\left[f_{i}\right]}\right)\right|_{x_{1}=0},
\end{aligned}
$$


where the first equality follows from the identity $\left(\begin{array}{c}i-j+1 \\ s\end{array}\right)=\left(\begin{array}{c}i-j \\ s\end{array}\right)+\left(\begin{array}{c}i-j \\ s-1\end{array}\right)$ of the binomial coefficients and the last equality follows from Lemma 3. Finally the cofactor expansion with respect to the first row gives the desired identity for (i).

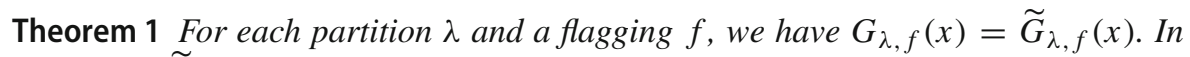
particular, $\widetilde{G}_{\lambda, f}(x)$ is a polynomial in $x$.

Proof By induction on $|f|:=f_{1}+\cdots+f_{r}$, Propositions 1 and 2 imply the claim: for the base case $|f|=0$, the claim holds trivially. If $f_{1}=1$, apply Proposition 2, and if $f_{1}>1$, apply Proposition 1 . In both cases, the claim follows from the induction hypothesis.

Remark 1 In the form of the determinant formula $\widetilde{G}_{\lambda, f}(x)$, it is now immediately clear that the flagged Grothendieck polynomials are actually polynomials in $x$ and $\beta$ since each entry of the determinant is a power series. Theorem 1 assures that $\widetilde{G}_{\lambda, f}(x)$ becomes a polynomial after it is expanded. This allows us to compute the flagged Grothendieck polynomials explicitly using the determinant formula by setting $\beta^{n}=0$ for sufficiently large $n$ so that each entry of the determinant is a finite sum. This partially answers to the question raised in [13, Question 2.4.2].

\section{Grothendieck polynomials}

In this section, we show that the Grothendieck polynomials associated with a vexillary permutation is a flagged Grothendieck polynomial, recovering the results of KnutsonMiller-Yong [15] and Hudson-Matsumura [12]. Based on Propositions 1 and 2, we also show that any flagged Grothendieck polynomial can be obtained from a monomial in the same way as any Grothendieck polynomial is defined. The proof in this section is completely combinatorial, while the original proofs in Knutson-Miller-Yong [15] and Hudson-Matsumura [12] are geometric. On the other hand, Knutson-Miller-Yong also gives a combinatorial proof of their tableau formula in [14] by showing that it satisfies Lascoux's transition formula for Grothendieck polynomials while ours is an alternative combinatorial proof using the compatibility of the tableau formula with the divided difference operators.

The Grothendieck polynomial $\mathcal{G}_{w}=\mathcal{G}_{w}\left(x_{1}, \ldots, x_{n}\right)$ associated with a permutation $w \in S_{n}$ is defined as follows. We use the same convention as in [4]. For the longest element $w_{0}$ in $S_{n}$, we set

$$
\mathcal{G}_{w_{0}}=x_{1}^{n-1} x_{2}^{n-2} \cdots x_{n-2}^{2} x_{n-1}=\prod_{i=1}^{n-1} x_{i}^{n-i}
$$

If $w$ is not the longest, we can find a positive integer $i$ such that $\ell\left(w s_{i}\right)=\ell(w)+1$ and then define

$$
\mathcal{G}_{w}:=\pi_{i}\left(\mathcal{G}_{w s_{i}}\right)
$$


This definition is independent of the choice of $s_{i}$ because the operators $\pi_{i}$ satisfy the Coxeter relations. By the same reason, we can define $\pi_{w}$ by $\pi_{w}=\pi_{i_{k}} \cdots \pi_{i_{1}}$ where $w=s_{i_{1}} \cdots s_{i_{k}}$ and $\ell(w)=k$.

Now we recall how to obtain a partition $\lambda(w)$ and a flagging $f(w)$ for each vexillary permutation $w \in S_{n}$. We follow [9,15] (cf. [12]). Let $r_{w}$ be the rank function of $w \in S_{n}$ defined by $r_{w}(p, q):=\sharp\{i \leq p \mid w(i) \leq q\}$, and we define the diagram $D(w)$ of $w$ by

$$
D(w):=\left\{(p, q) \in\{1, \ldots, n\} \times\{1, \ldots, n\} \mid \pi(p)>q \text {, and } \pi^{-1}(q)>p\right\} .
$$

We call an element of the grid $\{1, \ldots, n\} \times\{1, \ldots, n\}$ a box. The essential set $\mathcal{E} s s(w)$ of $w$ is the subset of $D(w)$ given by

$$
\mathcal{E} s s(w):=\{(p, q) \mid(p+1, q),(p, q+1) \notin D(w)\} .
$$

A permutation $w \in S_{n}$ is called vexillary if it avoids the pattern (2143), i.e., there is no $a<b<c<d$ such that $w(b)<w(a)<w(d)<w(c)$. In [20], a vexillary permutation was called a single-shape permutations. Fulton showed in [9] that $w \in S_{n}$ is vexillary if and only if the boxes in $\mathcal{E} s s(w)$ are placed along the direction going from northeast to southwest. We can assign a partition $\lambda(w)$ to each vexillary permutation $w$ as follows: Let the number of boxes $(i, i+k)$ in the $k$ th diagonal of the Young diagram of $\lambda(w)$ be equal to the number of boxes in the $k$ th diagonal of $D(w)$ for each $k$ (see $[14,15])$. This defines a bijection $\phi$ from $D(w)$ to $\lambda$, namely $\phi(p, q)=$ $\left(p-r_{w}(p, q), q-r_{w}(p, q)\right)$ for each $(p, q) \in D(w)$. In particular, $\phi$ restricted to $\mathcal{E} s s(w)$ is a bijection onto the set of the southeast corners of $\lambda(w)$. Let $r$ be the length of $\lambda(w)$. The flagging $f(w)=\left(f(w)_{1}, \ldots, f(w)_{r}\right)$ associated with $w$ is defined as follows. We can choose a subset $\left\{\left(p_{i}, q_{i}\right), i=1, \ldots, r\right\}$ of $\{1, \ldots, n\} \times\{1, \ldots, n\}$ containing $\mathcal{E} s s(w)$ and satisfying

$$
\begin{aligned}
& p_{1} \leq p_{2} \leq \cdots \leq p_{r}, \quad q_{1} \geq q_{2} \geq \cdots \geq q_{r} \\
& p_{i}-r_{w}\left(p_{i}, q_{i}\right)=i, \quad \forall i=1, \ldots, r
\end{aligned}
$$

In [12], we called this subset $\left\{\left(p_{i}, q_{i}\right)\right\}$ a flagging set of $w$ and used it to express the double Grothendieck polynomials as a determinant. We set $f(w)$ by letting $f(w)_{i}:=$ $p_{i}$. We can always express $\lambda(w)$ by $\lambda_{i}=q_{i}-p_{i}+i$ for each $i=1, \ldots, r$. Remark that the set $\operatorname{FSVT}(\lambda(w), f(w))$ does not depend on the choice of flagging sets.

Example 2 Consider a vexillary permutation $w=(w(1) \cdots w(5))=(23541)$ in $S_{5}$. The rank function $r_{w}$ of $w$ is indicated below as a matrix. The permutation matrix $M_{w}$ is defined by $\left(M_{w}\right)_{i j}=\delta_{w(i), j}$. In the picture below, we represent 1 in $M_{w}$ by a dot and the boxes in $D(w)$ by squares. We make hooks by drawing lines from each dot going south and east, and then $D(w)$ is the collection of boxes that are not on the hooks. We see that $\lambda(w)=\left(\lambda_{1}, \ldots, \lambda_{4}\right)=(2,1,1,1)$. For a flagging set of $w$, we must have $\left(p_{1}, q_{1}\right)=(3,4)$ and $\left(p_{4}, q_{4}\right)=(4,1)$ since they consist $\mathcal{E} s s(w)$. Then the conditions (3.1) and (3.2) require that we must choose $\left(p_{2}, q_{2}\right)$ from $(3,2)$ and 
$(4,3)$ and $\left(p_{3}, q_{3}\right)$ from $(3,1)$ and $(4,2)$ in such a way that $p_{2} \leq p_{3}$ and $q_{2} \geq q_{3}$. Thus $f(w)=(3,3,3,4),(3,3,4,4)$ or $(3,4,4,4)$. By the column strictness, each of the flaggings gives the same collection of flagging set-valued tableaux.

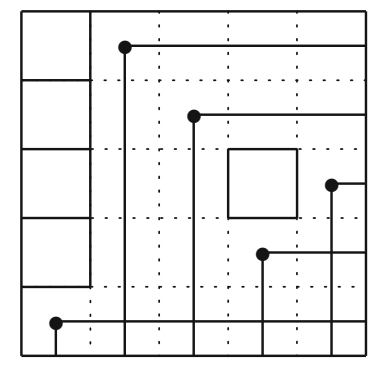

$$
r_{w}=\left[\begin{array}{lllll}
0 & 1 & 1 & 1 & 1 \\
0 & 1 & 2 & 2 & 2 \\
0 & 1 & 2 & 2 & 3 \\
0 & 1 & 2 & 3 & 4 \\
1 & 2 & 3 & 4 & 5
\end{array}\right]
$$

Theorem 2 If $w$ is a vexillary permutation, then $\mathcal{G}_{w}=G_{\lambda(w), f(w)}$.

Proof By Propositions 1 and 2, the proof is almost identical to the one given by Wachs for the flagged Schur functions in [20]. We write the proof for completeness since we have slightly different terminologies and notations.

First we observe that for the longest element $w_{0} \in S_{n}$, we have $\lambda\left(w_{0}\right)=(n-$ $1, n-2, \ldots, 1)$ and $f\left(w_{0}\right)=(1,2,3, \ldots, n-1)$. Therefore by definition we have

$$
G_{\lambda\left(w_{0}\right), f\left(w_{0}\right)}=\prod_{i=1}^{n-1} x_{i}^{n-i}=\mathcal{G}_{w_{0}} .
$$

We show the claim by induction on $\left(n, \ell\left(w_{0}\right)-\ell(w)\right)$ with the lexicographic order where $\ell(w)$ denotes the length $w \in S_{n}$. We say that $w$ has a descent at $i$ if $w_{i}>w_{i+1}$. Let $d$ be the leftmost descent of $w$. We consider the following three cases.

Case 1 Assume $d>1$. Let $w^{\prime}=w s_{d-1}$, then it is easy to see that $w^{\prime}$ is vexillary. Since $\ell\left(w^{\prime}\right)>\ell(w)$, we have $\mathcal{G}_{w^{\prime}}=G_{\lambda\left(w^{\prime}\right), f\left(w^{\prime}\right)}$ by the induction hypothesis. We can also observe that $\lambda\left(w^{\prime}\right)_{1}=\lambda(w)_{1}+1$ and $\lambda\left(w^{\prime}\right)_{i}=\lambda(w)_{i}$ for $i \geq 2$. Furthermore $f_{1}\left(w^{\prime}\right)=d-1=f_{1}(w)-1$ and $f\left(w^{\prime}\right)_{i}=f(w)_{i}$ for $i \geq 2$. Thus, by Proposition 1 , we have

$$
\mathcal{G}_{w}=\pi_{d-1} \mathcal{G}_{w^{\prime}}=\pi_{d-1} G_{\lambda\left(w^{\prime}\right), f\left(w^{\prime}\right)}=G_{\lambda(w), f(w)} .
$$

This completes the case $d>1$.

Case 2 Assume $d=1$ and $w(1)<n$. Let $w^{\prime}:=s_{w(1)} w$ so that $w^{\prime}(1)=w(1)+1$ and $w^{\prime}(l)=w(1)$ for $l$ such that $w(l)=w(1)+1$. It is clear that $w^{\prime}$ is vexillary. Since the leftmost descent of $w$ is 1 , we have $\ell\left(w^{\prime}\right)=\ell(w)+1$. Consider the vexillary permutation

$$
u=(w(1)+1, w(1), w(1)+2, w(1)+3, \ldots, n, w(1)-1, w(1)-2, \ldots, 1) .
$$

Since $w$ is vexillary, all numbers greater than $w(1)$ appear in ascending order in $w$, and therefore we can find integers $i_{1}, \ldots, i_{k}$ greater than 1 , satisfying $w^{\prime} s_{i_{1}} s_{i_{2}} \cdots s_{i_{k}}=u$ 
and $\ell\left(w^{\prime}\right)+k=\ell(u)$. For such a choice, we also have $w s_{i_{1}} s_{i_{2}} \cdots s_{i_{k}} s_{1}=u$. Thus by definition we have

$$
\mathcal{G}_{w}=\pi_{i_{1}} \cdots \pi_{i_{k}} \pi_{1} \mathcal{G}_{u} \text { and } \mathcal{G}_{w^{\prime}}=\pi_{i_{1}} \cdots \pi_{i_{k}} \mathcal{G}_{u}
$$

By induction, we have $\mathcal{G}_{u}=G_{\lambda(u), f(u)}$ and $\mathcal{G}_{w^{\prime}}=G_{\lambda\left(w^{\prime}\right), f\left(w^{\prime}\right)}$. We observe that

$$
\lambda(u)=(w(1), w(1)-1, w(1)-1, \ldots, w(1)-1, w(1)-2, w(1)-3, \ldots, 2,1)
$$

and $f(u)=(1,2,3, \ldots, n-1)$, from which we find

$$
\mathcal{G}_{u}=x_{1}^{w(1)} x_{2}^{w(1)-1} \cdots x_{n-w(1)+1}^{w(1)-1} x_{n-w(1)+2}^{w(1)-2} \cdots x_{n-2}^{2} x_{n-1}^{1} .
$$

(2.5) implies that $\pi_{1} \mathcal{G}_{u}=\left(1 / x_{1}\right) \mathcal{G}_{u}$. Since $i_{1}, \ldots, i_{k}$ are greater than 1 , again (2.5) implies that

$$
\mathcal{G}_{w}=\pi_{i_{1}} \cdots \pi_{i_{k}}\left(\left(1 / x_{1}\right) \mathcal{G}_{u}\right)=\left(1 / x_{1}\right) \mathcal{G}_{w}^{\prime}=\left(1 / x_{1}\right) G_{\lambda\left(w^{\prime}\right), f\left(w^{\prime}\right)}
$$

Furthermore, we observe that

$$
\begin{gathered}
\lambda\left(w^{\prime}\right)_{1}=\lambda(w)_{1}+1, \quad \lambda\left(w^{\prime}\right)_{i}=\lambda(w)_{i}, \quad \forall i=2, \ldots, r \\
f\left(w^{\prime}\right)_{1}=f(w)_{1}=1, \quad f\left(w^{\prime}\right)_{i}=f(w)_{i}, \quad \forall i=2, \ldots, r .
\end{gathered}
$$

This implies that $G_{\lambda(w), f(w)}=\left(1 / x_{1}\right) G_{\lambda\left(w^{\prime}\right), f(w)}$. Therefore we conclude that $\mathcal{G}_{w}=$ $G_{\lambda(w), f(w)}$.

Case 3 Assume $d=1$ and $w(1)=n$. Let $w_{0} w=s_{i_{1}} \cdots s_{i_{k}}$ where $\ell(w)+k=$ $\ell\left(w_{0}\right)$. Since $w(1)=n$, it follows that $i_{1}, \ldots, i_{k}$ are greater than 1 . Consequently,

$$
\begin{aligned}
\mathcal{G}_{w} & =\pi_{i_{k}} \pi_{i_{k-1}} \cdots \pi_{i_{1}}\left(x_{1}^{n-1} x_{2}^{n-2} \cdots x_{n-2}^{2} x_{n-1}^{1}\right) \\
& =x_{1}^{n-1} \pi_{i_{k}} \pi_{i_{k-1}} \cdots \pi_{i_{1}}\left(x_{2}^{n-2} \cdots x_{n-2}^{2} x_{n-1}^{1}\right) .
\end{aligned}
$$

Consider the vexillary permutation $w^{\prime}:=(w(2), \ldots, w(n))$ in $S_{n-1}$. By induction, we have $\mathcal{G}_{w^{\prime}}=G_{\lambda\left(w^{\prime}\right), f\left(w^{\prime}\right)}$. Moreover, by definition we have

$$
\pi_{i_{k}} \pi_{i_{k-1}} \cdots \pi_{i_{1}}\left(x_{2}^{n-2} \cdots x_{n-2}^{2} x_{n-1}^{1}\right)=\left(\mathcal{G}_{w^{\prime}}\right)^{\dagger}
$$

where $f(x)^{\dagger}$ denotes the function obtained from $f(x)$ by replacing $x_{i}$ with $x_{i+1}$. Thus $\mathcal{G}_{w}=x_{1}^{n-1}\left(\mathcal{G}_{w^{\prime}}\right)^{\dagger}$. Since $f\left(w^{\prime}\right)=\left(f\left(w^{\prime}\right)_{1}, \ldots, f\left(w^{\prime}\right)_{n-1}\right)=\left(f(w)_{2}-\right.$ $\left.1, \ldots, f(w)_{n}-1\right)$, it follows that

$$
\left(G_{\lambda\left(w^{\prime}\right), f\left(w^{\prime}\right)}\right)^{\dagger}=\left.G_{\lambda\left(w^{\prime}\right), \widetilde{f(w)}}\right|_{x_{1}=0},
$$


where $\widetilde{f(w)}=\left(f_{2}(w), \ldots, f_{n}(w)\right)$. Thus

$$
\mathcal{G}_{w}=x_{1}^{n-1}\left(G_{\lambda\left(w^{\prime}\right), f\left(w^{\prime}\right)}\right)^{\dagger}=x_{1}^{n-1} \cdot\left(\left.G_{\lambda\left(w^{\prime}\right), \widetilde{f(w)}}\right|_{x_{1}=0}\right) .
$$

where the right-hand side equals to $G_{\lambda(w), f(w)}$ by Proposition 2 . This completes the proof.

Theorem 3 Let $\lambda$ be a partition of length $r$ with a flagging $f$. If $f_{i}>i-1$ for each $i=1, \ldots, r$, then $G_{\lambda, f}$ is equal to $\pi_{w}\left(x_{1}^{a_{1}} \cdots x_{r}^{a_{r}}\right)$ where $a_{i}=\lambda_{i}+f_{i}-i$ and

$$
w=\left(s_{r} s_{r+1} \cdots s_{f_{r}-1}\right)\left(s_{r-1} s_{r} \cdots s_{f_{r-1}-1}\right) \cdots\left(s_{1} s_{2} \cdots s_{f_{1}-1}\right) .
$$

Otherwise, we have $G_{\lambda, f}=0$.

Proof First observe that if there is $k$ such that $f_{k} \leq k-1$, then $G_{\lambda, f}=0$ by the column strictness of the flagged set-valued tableaux. We assume that $f_{i}>i-1$ for each $i=1, \ldots, r$. We prove by induction on the sum of the flagging $|f|=$ $f_{1}+\cdots+f_{r}$. If $|f|=1$, then $f_{1}=1$ and $r=1$. In this case, we have $a_{1}=\lambda_{1}$ and $w=$ id so that $G_{\lambda, f}=x_{1}^{\lambda_{1}}=\pi_{\text {id }} x_{1}^{a_{1}}$. Assume that $|f|>1$. If $f_{1}>1$, consider $\lambda^{\prime}=\left(\lambda_{1}+1, \lambda_{2}, \ldots, \lambda_{r}\right)$ and $f^{\prime}=\left(f_{1}-1, f_{2}, \ldots, f_{r}\right)$. The induction hypothesis implies that $G_{\lambda^{\prime}, f^{\prime}}=\pi_{w s_{f_{1}-1}}\left(x_{1}^{a_{1}} \cdots x_{r}^{a_{r}}\right)$. By Proposition 1 and the Coxeter relation of divided differences, we obtain

$$
G_{\lambda, f}=\pi_{f_{1}-1} G_{\lambda^{\prime}, f^{\prime}}=\pi_{f_{1}-1} \pi_{w s_{f_{1}-1}}\left(x_{1}^{a_{1}} \cdots x_{r}^{a_{r}}\right)=\pi_{w}\left(x_{1}^{a_{1}} \cdots x_{r}^{a_{r}}\right) .
$$

If $f_{1}=1$, consider $\lambda^{\prime}=\left(\lambda_{2}, \ldots, \lambda_{r}\right)$ and $f^{\prime}=\left(f_{2}-1, \ldots, f_{r}-1\right)$. Then by definition we have $G_{\lambda, f}=x_{1}^{a_{1}}\left(G_{\lambda^{\prime}, f^{\prime}}\right)^{\dagger}$ where $f(x)^{\dagger}$ denotes the function obtained from $f(x)$ by replacing $x_{i}$ with $x_{i+1}$ as before and $a_{1}=\lambda_{1}$. On the other hand, by induction, we have $G_{\lambda^{\prime}, f^{\prime}}=\pi_{w^{\prime}}\left(x_{1}^{a_{1}^{\prime}} \cdots x_{r-1}^{a_{r-1}^{\prime}}\right)$ where $a_{i}^{\prime}=a_{i+1}$ and $w^{\prime}=s_{r-1} s_{r} \cdots s_{f_{r}-2} s_{r-2} s_{r-1} \cdots s_{f_{r-1}-2} \cdots s_{1} s_{2} \cdots s_{f_{2}-2}$. We can see that $\left(\pi_{w^{\prime}}\left(x_{1}^{a_{1}^{\prime}} \cdots x_{r-1}^{a_{r-1}^{\prime}}\right)\right)^{\dagger}=\pi_{w}\left(x_{2}^{a_{2}} \cdots x_{r}^{a_{r}}\right)$. Thus, since none of the transpositions in the expression of $w$ is equal to $s_{1}$, we have

$G_{\lambda, f}=x_{1}^{a_{1}} \pi_{w}\left(x_{2}^{a_{2}} \cdots x_{r}^{a_{r}}\right)=\pi_{w}\left(x_{1}^{a_{1}} x_{2}^{a_{2}} \cdots x_{r}^{a_{r}}\right)$.

\section{Flagged skew Grothendieck polynomials}

In this section, we introduced flagged skew Grothendieck polynomials and show the equivalence of their tableaux formula and determinant formula (Theorem 4). We follow Section 3 of [20]. Although the result in this section generalizes Theorem 1, the proof is essentially different since by considering a more general family of polynomials defined by flagged skew tableaux one manages to prove the equivalence inductively without using the compatibility with the divided difference operators. 
Consider two partitions $\lambda=\left(\lambda_{1} \geq \cdots \geq \lambda_{r}>0\right)$ and $\mu=\left(\mu_{1} \geq \cdots \geq \mu_{r} \geq 0\right)$ such that $\mu_{i} \leq \lambda_{i}$ for all $i=1, \ldots, r$ and two sequence of positive integers $f=$ $\left(f_{1}, \ldots, f_{r}\right)$ and $g=\left(g_{1}, \cdots, g_{r}\right)$ such that

$$
g_{i} \leq g_{i+1}, \quad \text { and } f_{i} \leq f_{i+1} \text {, whenever } \mu_{i}<\lambda_{i+1}
$$

In this case, we say that $f / g$ is a flagging of the skew shape $\lambda / \mu$. A flagged skew setvalued tableau of skew shape $\lambda / \mu$ with a flagging $f / g$ is a set-valued tableau of the skew shape $\lambda / \mu$ such that each filling in the $i$ th row is a subset of $\left\{g_{i}, g_{i}+1, \ldots, f_{i}\right\}$. We set $|\lambda / \mu|=|\lambda|-|\mu|$. Let $F S V T(\lambda / \mu, f / g)$ denote the set of all flagged skew tableaux of shape $\lambda / \mu$ with a flagging $f / g$. If $g=(1, \ldots, 1)$ and $f_{1}=\cdots=f_{r}$, then the associated flagged skew set-valued tableaux are nothing but the set-valued tableaux of skew shape $\lambda / \mu$ considered by Buch in [4].

Example 3 Consider the following skew shapes $\lambda / \mu$ with flagging $f / g$.

$-\lambda=(3,2), \mu=(1,0), f=(2,4)$, and $g=(1,2)$. The following tableaux, for example, are in $\operatorname{FSVT}(\lambda / \mu, f / g)$.
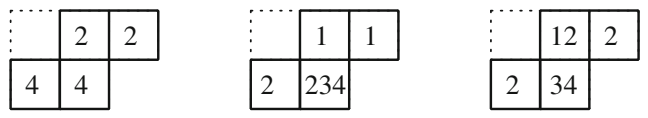

$-\lambda=(4,3,1,1), \mu=(2,2,1,0), f=(2,4,2,1)$, and $g=(1,2,3,1)$. The following tableaux, for example, are in $\operatorname{FSVT}(\lambda / \mu, f / g)$.
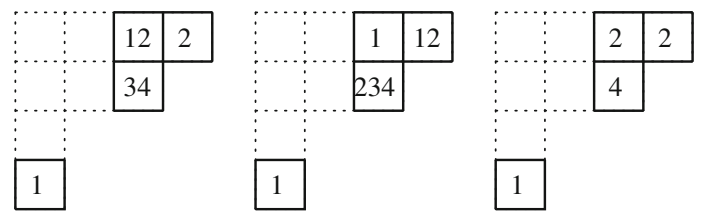

For a tableau $T$, let $x^{T}$ be the monomial in which the exponent of $x_{i}$ is the number of boxes in $T$ containing the integer $i$ and $|T|$ its total degree as before. We define the flagged skew Grothendieck polynomial of skew shape $\lambda / \mu$ with flagging $f / g$ by

$$
G_{\lambda / \mu, f / g}(x):=\sum_{T \in F S V T(\lambda / \mu, f / g)} \beta^{|T|-|\lambda / \mu|} x^{T} .
$$

We also define an element $\widetilde{G}_{\lambda / \mu, f / g}=\widetilde{G}_{\lambda / \mu, f / g}(x)$ of $\mathbb{Z}[\beta][[x]]$ by

$$
\widetilde{G}_{\lambda / \mu, f / g}(x):=\operatorname{det}\left(\sum_{s \geq 0}\left(\begin{array}{c}
i-j \\
s
\end{array}\right) \beta^{s} G_{\lambda_{i}-\mu_{j}+j-i+s}^{\left[f_{i} / g_{j}\right]}\right)_{1 \leq i, j \leq r} .
$$


where for $p, q \in \mathbb{N}$, the function $G_{m}^{[p / q]}=G_{m}^{[p / q]}(x)$ is defined by the generating function

$$
\sum_{m \in \mathbb{Z}} G_{m}^{[p / q]} u^{m}=\frac{1}{1+\beta^{-1} u} \prod_{q \leq i \leq p} \frac{1+\beta x_{i}}{1-x_{i} u}
$$

Note that $G_{-m}^{[p / q]}=(-\beta)^{m}$ for all integer $m \geq 0$ and, if $q>p$, then $G_{m}^{[p / q]}=0$ for all $m>0$.

The goal of this section is to show $G_{\lambda / \mu, f / g}(x)=\widetilde{G}_{\lambda / \mu, f / g}(x)$ (Theorem4). If we specialize at $\beta=0, G_{\lambda / \mu, f / g}(x)$ becomes the (row) flagged skew Schur polynomial in [20]. Furthermore, under this specialization, $\widetilde{G}_{\lambda / \mu, f / g}(x)$ gives nothing but the corresponding Jacobi-Trudi formula also in [20], since $G_{m}^{[p / q]}(x)$ becomes the complete symmetric function of degree $m$ in variables $x_{q}, x_{q+1}, \ldots, x_{p}$.

First of all, we show the following basic formulas for the elements $G_{m}^{[p / q]}$.

Lemma 4 If $q \leq p$, we have

$$
\begin{aligned}
G_{m}^{[p / q]} & =x_{q} G_{m-1}^{[p / q]}+\left(1+\beta x_{q}\right) G_{m}^{[p / q+1]}, \\
G_{m-1}^{[p / q]}+\beta G_{m}^{[p / q+1]} & =\frac{1}{1+\beta x_{q}}\left(G_{m-1}^{[p / q]}+\beta G_{m}^{[p / q]}\right),
\end{aligned}
$$

for each $m \in \mathbb{Z}$.

Proof Equation (4.2) follows by comparing the coefficient of $u^{m}$ in the identity

$$
\left(1-x_{q} u\right) \sum_{m \in \mathbb{Z}} G_{m}^{[p / q]} u^{m}=\left(1+\beta x_{q}\right) \sum_{m \in \mathbb{Z}} G_{m}^{[p / q+1]} u^{m} .
$$

For (4.3), we compute the generating function of $G_{m-1}^{[p / q]}+\beta G_{m}^{[p / q+1]}$ :

$$
\begin{aligned}
& \sum_{m \in \mathbb{Z}}\left(G_{m-1}^{[p / q]}+\beta G_{m}^{[p / q+1]}\right) u^{m} \\
& =u \frac{1}{1+\beta u^{-1}} \prod_{q \leq i \leq p} \frac{1+\beta x_{i}}{1-x_{i} u}+\beta \frac{1}{1+\beta u^{-1}} \prod_{q+1 \leq i \leq p} \frac{1+\beta x_{i}}{1-x_{i} u} \\
& =\frac{1}{1+\beta u^{-1}} \prod_{q \leq i \leq p} \frac{1+\beta x_{i}}{1-x_{i} u}\left(u+\beta \frac{1-x_{q} u}{1+\beta x_{q}}\right) \\
& =\left(\sum_{m \in \mathbb{Z}} G_{m}^{[p / q]} u^{m}\right)\left(\frac{u+\beta}{1+\beta x_{q}}\right) \\
& =\frac{1}{1+\beta x_{q}}\left(\sum_{m \in \mathbb{Z}}\left(G_{m-1}^{[p / q]}+\beta G_{m}^{[p / q]}\right) u^{m}\right)
\end{aligned}
$$

Thus by comparing the coefficients of $u^{m}$, we find that (4.3) holds. 
The following four propositions will be used for the proof of Theorem 4 .

Proposition 3 Let $k$ be such that $\mu_{k} \geq \lambda_{k+1}$. Then we have

(i) $\widetilde{G}_{\lambda / \mu, f / g}=\widetilde{G}_{\hat{\lambda} / \hat{\mu}, \hat{f} / \hat{g}} \cdot \widetilde{G}_{\check{\lambda} / \check{\mu}, \check{f} / \check{g}}$.

(ii) $G_{\lambda / \mu, f / g}=G_{\hat{\lambda} / \hat{\mu}, \hat{f} / \hat{g}} \cdot G_{\check{\lambda} / \check{\mu}, \check{f} / \check{g}}$.

where $\wedge$ and ${ }^{\vee}$ applied to a sequence $\left(t_{1}, \ldots, t_{r}\right)$ denote the sequences $\left(t_{1}, \ldots, t_{k}\right)$ and $\left(t_{k+1}, \ldots, t_{r}\right)$, respectively.

Proof The identity (ii) is trivial from the definition. We prove (i). If $j \leq k<i$, we have $\lambda_{i}-\mu_{j} \leq 0$. This implies that $(i, j)$-entry of the determinant of $\widetilde{G}_{\lambda / \mu, f / g}$ is 0 for $j \leq k<i$. Indeed, since $G_{-m}^{[p / q]}=(-\beta)^{m}$ for $m \geq 0$ and by an identity of binomial coefficients, we have

$$
\begin{aligned}
\sum_{s \geq 0}\left(\begin{array}{c}
i-j \\
s
\end{array}\right) \beta^{s} G_{\lambda_{i}-\mu_{j}+j-i+s}^{\left[f_{i} / g_{j}\right]} & =\sum_{0 \leq s \leq i-j}\left(\begin{array}{c}
i-j \\
s
\end{array}\right) \beta^{s}(-\beta)^{-\left(\lambda_{i}-\mu_{j}+j-i+s\right)} \\
& =(-\beta)^{-\left(\lambda_{i}-\mu_{j}+j-i\right)} \sum_{0 \leq s \leq i-j}(-1)^{s}\left(\begin{array}{c}
i-j \\
s
\end{array}\right) \\
& =0 .
\end{aligned}
$$

Thus the determinant of $\widetilde{G}_{\lambda / \mu, f / g}$ is the product of the determinants of $\widetilde{G}_{\hat{\lambda} / \hat{\mu}, \hat{f} / \hat{g}}$ and

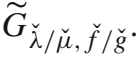

Proposition 4 Let $k$ be such that $\mu_{k}<\lambda_{k}$ and $g_{k} \leq f_{k}$. If $g_{k}<g_{k+1}($ or $k=r)$ and $\mu_{k-1}>\mu_{k}($ or $k=1)$, then we have

(i) $\widetilde{G}_{\lambda / \mu, f / g}=x_{g_{k}} \widetilde{G}_{\lambda / \mu^{\prime}, f / g}+\left(1+\beta x_{g_{k}}\right) \widetilde{G}_{\lambda / \mu, f / g^{\prime}}$,

(ii) $G_{\lambda / \mu, f / g}=x_{g_{k}} G_{\lambda / \mu^{\prime}, f / g}+\left(1+\beta x_{g_{k}}\right) G_{\lambda / \mu, f / g^{\prime}}$,

where the symbol' applied to a sequence denotes adding 1 to the kth element of the sequence.

Proof The assumption guarantees that $f / g$ and $f / g^{\prime}$ are flaggings of the skew shapes $\lambda / \mu^{\prime}$ and $\lambda / \mu$, respectively. First we prove (i). The columns in the determinants of both sides are identical except for the $k$ th one. Thus the equality (i) holds if, for all $i=1, \ldots, r$, we have

$$
\begin{aligned}
& \sum_{s \geq 0}\left(\begin{array}{c}
i-k \\
s
\end{array}\right) \beta^{s} G_{\lambda_{i}-\mu_{k}+k-i+s}^{\left[f_{i} / g_{k}\right]} \\
& =x_{g_{k}} \sum_{s \geq 0}\left(\begin{array}{c}
i-k \\
s
\end{array}\right) \beta^{s} G_{\lambda_{i}-\mu_{k}-1+k-i+s}^{\left[f_{i} / g_{k}\right]} \\
& \quad+\left(1+\beta x_{g_{k}}\right) \sum_{s \geq 0}\left(\begin{array}{c}
i-k \\
s
\end{array}\right) \beta^{s} G_{\lambda_{i}-\mu_{k}+k-i+s}^{\left[f_{i} / g_{k}+1\right]} .
\end{aligned}
$$

If $g_{k} \leq f_{i}$, Eq. (4.2) proves the claim. Suppose that $g_{k}>f_{i}$. This implies that $f_{i}<f_{k}$ and $i \neq k$. If $i<k$, then since $\mu_{k}<\lambda_{k} \leq \lambda_{i}$, we have $\lambda_{i}-\mu_{k}+k-i \geq 2$. In this 
case, the both sides of (4.4) are zero since $G_{m}^{\left[f_{i} / g_{k}\right]}=0$ for all $m>0$. Thus it remains to show (4.4) for the case when $i>k$ and $\lambda_{i}-\mu_{k}+k-i \leq 1$. Since $f_{i}<f_{k}$, the condition (4.1) implies $\mu_{k} \geq \lambda_{i}$. Now we claim that (4.4) follows by evaluating the right-hand side using the identity $G_{-m}^{\left[f_{i} / g_{k}\right]}=(-\beta)^{m}$ for all $m \geq 0$. Indeed, let $a:=i-k>0$ and $b:=\mu_{k}-\lambda_{i} \geq 0$, then we have

$$
\begin{aligned}
& x_{g_{k}} \sum_{s \geq 0}\left(\begin{array}{l}
a \\
s
\end{array}\right) \beta^{s} G_{-b-1-a+s}^{\left[f_{i} / g_{k}\right]}+\left(1+\beta x_{g_{k}}\right) \sum_{s \geq 0}\left(\begin{array}{l}
a \\
s
\end{array}\right) \beta^{s} G_{-b-a+s}^{\left[f_{i} / g_{k}+1\right]} \\
& =x_{g_{k}} \sum_{0 \leq s \leq a}\left(\begin{array}{l}
a \\
s
\end{array}\right) \beta^{s}(-\beta)^{b+1+a-s}+\left(1+\beta x_{g_{k}}\right) \sum_{0 \leq s \leq a}\left(\begin{array}{l}
a \\
s
\end{array}\right) \beta^{s}(-\beta)^{b+a-s} \\
& =\sum_{0 \leq s \leq a}\left(\begin{array}{l}
a \\
s
\end{array}\right) \beta^{s}(-\beta)^{b+a-s} \\
& =\sum_{s \geq 0}\left(\begin{array}{l}
a \\
s
\end{array}\right) \beta^{s} G_{-b-a+s}^{\left[f_{i} / g_{k}\right]} .
\end{aligned}
$$

This finishes the proof of (i).

To prove (ii), we partition the set $\operatorname{FSVT}(\lambda / \mu, f / g)$ into three subsets $\mathcal{F}_{1}, \mathcal{F}_{2}$ and $\mathcal{F}_{3}: \mathcal{F}_{1}$ consists of those tableaux such that the filling in the leftmost box in the $k$ th row is exactly $\left\{g_{k}\right\}, \mathcal{F}_{2}$ consists of those tableaux such that the filling in the leftmost box in the $k$ th row does not contain $g_{k}$, and $\mathcal{F}_{3}$ consists of the rest. Note that by the condition (4.1) the set $\mathcal{F}_{1}$ is non-empty. Since $g_{k+1}>g_{k}$, there is a bijection from $\mathcal{F}_{1}$ to $\operatorname{FSVT}\left(\lambda / \mu^{\prime}, f / g\right)$ sending $T$ to $T^{\prime}$ obtained from removing the leftmost box together with its filling $\left\{g_{k}\right\}$ from the $k$ th row. Thus $\sum_{T \in \mathcal{F}_{1}} \beta^{|T|-|\lambda / \mu|} x^{T}=x_{g_{k}} G_{\lambda / \mu^{\prime}, f / g}$. It is clear that $\mathcal{F}_{2}=\operatorname{FSVT}\left(\lambda / \mu, f / g^{\prime}\right)$ so that $\sum_{T \in \mathcal{F}_{2}} \beta^{|T|-|\lambda / \mu|} x^{T}=G_{\lambda / \mu, f / g^{\prime}}$. Furthermore, since $g_{k+1}>g_{k}$, we have a bijection from $\mathcal{F}_{3}$ to $\operatorname{FSVT}\left(\lambda / \mu, f / g^{\prime}\right)$ by removing the entry $g_{k}$ from the $k$ th row and leaving the rest unchanged. Then it follows that $\sum_{T \in \mathcal{F}_{3}} \beta^{|T|-|\lambda / \mu|} x^{T}=\beta x_{g_{k}} G_{\lambda / \mu, f / g^{\prime}}$. This proves (ii).

Proposition 5 Let $k$ be such that $g_{k-1}=g_{k}$ and $\mu_{k-1}=\mu_{k}$. Then

(i) $\widetilde{G}_{\lambda / \mu, f / g}=\widetilde{G}_{\lambda / \mu, f / g^{\prime}}$,

(ii) $G_{\lambda / \mu, f / g}=G_{\lambda / \mu, f / g^{\prime}}$,

where the symbol' is as in Proposition 4.

Proof For (i), first we see that the determinants in the equation are identical except for the $k$ th column. We compute the difference of their $(i, k)$-entries by using (4.2) and (4.3):

$$
\begin{aligned}
& \sum_{s \geq 0}\left(\begin{array}{c}
i-k \\
s
\end{array}\right) \beta^{s} G_{\lambda_{i}-\mu_{k}+k-i+s}^{\left[f_{i} / g_{k}\right]}-\sum_{s \geq 0}\left(\begin{array}{c}
i-k \\
s
\end{array}\right) \beta^{s} G_{\lambda_{i}-\mu_{k}+k-i+s}^{\left[f_{i} / g_{k}+1\right]} \\
& =\sum_{s \geq 0}\left(\begin{array}{c}
i-k \\
s
\end{array}\right) \beta^{s}\left(x_{g_{k}} G_{\lambda_{i}-\mu_{k}+k-i+s-1}^{\left[f_{i} / g_{k}\right]}+\beta x_{g_{k}} G_{\lambda_{i}-\mu_{k}+k-i+s}^{\left[f_{i} / g_{k}+1\right]}\right)
\end{aligned}
$$




$$
\begin{aligned}
& =\frac{x_{g_{k}}}{1+\beta x_{g_{k}}} \sum_{s \geq 0}\left(\begin{array}{c}
i-k \\
s
\end{array}\right) \beta^{s}\left(G_{\lambda_{i}-\mu_{k}+k-i+s-1}^{\left[f_{i} / g_{k}\right]}+\beta G_{\lambda_{i}-\mu_{k}+k-i+s}^{\left[f_{i} / g_{k}\right]}\right) \\
& =\frac{x_{g_{k}}}{1+\beta x_{g_{k}}} \sum_{s \geq 0}\left(\left(\begin{array}{c}
i-k \\
s
\end{array}\right)+\left(\begin{array}{c}
i-k \\
s-1
\end{array}\right)\right) \beta^{s} G_{\lambda_{i}-\mu_{k}+k-i+s-1}^{\left[f_{i} / g_{k}\right]} \\
& =\frac{x_{g_{k}}}{1+\beta x_{g_{k}}} \sum_{s \geq 0}\left(\begin{array}{c}
i-k+1 \\
s
\end{array}\right) \beta^{s} G_{\lambda_{i}-\mu_{k}+k-i+s-1}^{\left[f_{i} / g_{k}\right]} .
\end{aligned}
$$

Here the first equality by (4.2), the second is by (4.3), and the last follows from the identity of the binomial coefficients $\left(\begin{array}{l}n \\ s\end{array}\right)+\left(\begin{array}{c}n \\ s-1\end{array}\right)=\left(\begin{array}{c}n+1 \\ s\end{array}\right)$ for $n, s \in \mathbb{Z}$. This shows that in the determinant $\widetilde{G}_{\lambda / \mu, f / g}-\widetilde{G}_{\lambda / \mu, f / g^{\prime}}$, the $k$ th column coincides with $\frac{x_{g_{k}}}{1+\beta x_{g_{k}}}$ times the $(k-1)$-st column. Thus $\widetilde{G}_{\lambda / \mu, f / g}-\widetilde{G}_{\lambda / \mu, f / g^{\prime}}=0$.

For (ii), it suffices to observe that $F S V T(\lambda / \mu, f / g)=F S V T\left(\lambda / \mu, f / g^{\prime}\right)$ from the column strictness of the flagged skew set-valued tableaux.

Proposition 6 If $f_{k}<g_{k}$ and $\mu_{k}<\lambda_{k}$ for some $k$, then

(ii) $\widetilde{G}_{\lambda / \mu, f / g}=0$,

(ii) $G_{\lambda / \mu, f / g}=0$.

Proof For (ii), it suffices to observe that $\operatorname{FSVT}(\lambda / \mu, f / g)=\varnothing$. We prove (i). If $r=1$, then $k=1$ and $\widetilde{G}_{\lambda / \mu, f / g}(x)=G_{\lambda_{1}-\mu_{1}}^{\left[f_{1} / g_{1}\right]}=0$. Suppose $r>1$. If there is $i$ such that $\mu_{i} \geq \lambda_{i+1}$, then the claim follows from Proposition 3 and the induction hypothesis. Suppose that $\mu_{i}<\lambda_{i+1}$ for all $i$. In this case, (4.1) and the assumption imply that $\mu_{i}<\lambda_{j}$ and $f_{i}<g_{j}$ for all $i \leq k \leq j$. Then we can see that the $(i, j)$-entry of the determinant of $\widetilde{G}_{\lambda / \mu, f / g}$ is 0 for all $i$ and $j$ such that $i \leq k \leq j$ since $G_{m}^{\left[f_{i} / g_{j}\right]}=0$ for all $m>0$. Thus the claim follows.

Theorem 4 We have $\widetilde{G}_{\lambda / \mu, f / g}(x)=G_{\lambda / \mu, f / g}(x)$.

Proof With the help of Propositions 3-6, the proof is exactly the same as the one for Theorem 3.5 [20]. We write the proof below for completeness. We prove this by induction on $(r, \lambda-\mu, f-g)$ ordered lexicographically where $r$ is the length of the partition $\lambda$. If $r=1, \widetilde{G}_{\lambda / \mu, f / g}$ and $G_{\lambda / \mu, f / g}$ are the same one row Grothendieck polynomial of degree $\lambda_{1}-\mu_{1}$ with the shifted variables, thus the claim holds. Suppose $r>1$. If $\lambda_{i}-\mu_{i}=0$ for some $i$, then we have $\lambda_{i+1} \leq \lambda_{i}=\mu_{i}$ or $\lambda_{i}=\mu_{i} \leq \mu_{i-1}$. We apply Proposition 3 for $k=i-1$ or $i$, and then the claim follows from the induction hypothesis. Suppose $\lambda_{i}-\mu_{i}>0$ for all $i=1, \ldots, r$. If $f_{k}-g_{k}<0$ for some $k$, then the claim follows from Proposition 6. Thus suppose further that $f_{i}-g_{i} \geq 0$ for all $i=1, \ldots, r$. Let $k$ be such that $g_{1} \geq g_{2} \geq \cdots \geq g_{k}<g_{k+1}$ (or set $k=r$ ). If $\mu_{k-1}>\mu_{k}$ (or $k=1$ ), we can apply Proposition 4 and the claim follows by induction. If $\mu_{k-1}=\mu_{k}$, then $\mu_{k-1} \leq \lambda_{k}$ and hence $g_{k-1} \leq g_{k}$. This implies that $g_{k-1}=g_{k}$. Now we can apply Proposition 5, and the claim follows from the induction hypothesis.

Acknowledgements The author would like to thank Takeshi Ikeda for useful discussions. 


\section{References}

1. Anderson, D.: K-theoretic Chern class formulas for vexillary degeneracy loci. ArXiv e-prints (2017)

2. Anderson, D., Chen, L., Tarasca, N.: K-classes of Brill-Noether loci and a determinantal formula. ArXiv e-prints (2017)

3. Billey, S.C., Jockusch, W., Stanley, R.P.: Some combinatorial properties of Schubert polynomials. J. Algebraic Combin. 2(4), 345-374 (1993)

4. Buch, A.S.: A Littlewood-Richardson rule for the $K$-theory of Grassmannians. Acta Math. 189(1), 37-78 (2002)

5. Chen, W.Y.C., Li, B., Louck, J.D.: The flagged double Schur function. J. Algebraic Combin. 15(1), 7-26 (2002)

6. Chen, W.Y.C., Yan, G.G., Yang, A.L.B.: The skew Schubert polynomials. European J. Combin. 25(8), 1181-1196 (2004)

7. Fomin, S., Kirillov, A.N.: The Yang-Baxter equation, symmetric functions, and Schubert polynomials. Discrete Math. 153(1-3), 123-143 (1996). In: Proceedings of the 5th Conference on Formal Power Series and Algebraic Combinatorics (Florence, 1993)

8. Fomin, S., Kirillov, A.N.: Grothendieck polynomials and the Yang-Baxter equation. In: Formal power series and algebraic combinatorics/Séries formelles et combinatoire algébrique, pp. 183-189. DIMACS, Piscataway, NJ (sd)

9. Fulton, W.: Flags, Schubert polynomials, degeneracy loci, and determinantal formulas. Duke Math. J. 65(3), 381-420 (1992)

10. Hudson, T., Ikeda, T., Matsumura, T., Naruse, H.: Degeneracy loci classes in $K$-theory-determinantal and Pfaffian formula. Adv. Math. 320, 115-156 (2017)

11. Hudson, T., Matsumura, T.: Segre classes and Kempf-Laksov formula in algebraic cobordism. ArXiv e-prints (2016)

12. Hudson, T., Matsumura, T.: Vexillary degeneracy loci classes in $K$-theory and algebraic cobordism. European J. Combin. 70, 190-201 (2018)

13. Knutson, A., Miller, E.: Gröbner geometry of Schubert polynomials. Ann. of Math. (2) 161(3), 12451318 (2005)

14. Knutson, A., Miller, E., Yong, A.: Tableau complexes. Israel J. Math. 163, 317-343 (2008)

15. Knutson, A., Miller, E., Yong, A.: Gröbner geometry of vertex decompositions and of flagged tableaux. J. Reine Angew. Math. 630, 1-31 (2009)

16. Lascoux, A.: Anneau de Grothendieck de la variété de drapeaux. In: Cartier, P., Illusie, L., Katz, N.M., Laumon, G., Manin, Y.I., Ribet, K.A. (eds.) The Grothendieck Festschrift, Vol. III, Progress in Mathematics, vol. 88, pp. 1-34. Birkhäuser Boston, Boston (1990)

17. Lascoux, A., Schützenberger, M.P.: Polynômes de Schubert. C. R. Acad. Sci. Paris Sér. I Math. 294(13), 447-450 (1982)

18. Lascoux, A., Schützenberger, M.P.: Structure de Hopf de l'anneau de cohomologie et de l'anneau de Grothendieck d'une variété de drapeaux. C. R. Acad. Sci. Paris Sér. I Math. 295(11), 629-633 (1982)

19. Matsumura, T.: An algebraic proof of determinant formulas of Grothendieck polynomials. Proc. Japan Acad. Ser. A Math. Sci. 93(8), 82-85 (2017)

20. Wachs, M.L.: Flagged Schur functions, Schubert polynomials, and symmetrizing operators. J. Combin. Theory Ser. A 40(2), 276-289 (1985) 